\title{
Raloxifene reduces triple-negative breast cancer tumor growth and decreases EGFR expression
}

\author{
SEBASTIEN TAURIN, KIRSTIE M. ALLEN, MARISSA J. SCANDLYN and RHONDA J. ROSENGREN
}

\begin{abstract}
Department of Pharmacology and Toxicology, University of Otago, Adams Building, Dunedin 9016, New Zealand
\end{abstract}
Received April 21, 2013; Accepted June 3, 2013

DOI: $10.3892 /$ ijo.2013.2012

\begin{abstract}
The poor prognosis of patients with triple-negative breast cancer (TNBC) and the lack of targeted treatments have raised the need for alternative therapies. Previous studies have suggested an effect of raloxifene, a selective estrogen receptor modulator that is independent of the estrogen receptor (ER). Therefore, we assessed the therapeutic value of raloxifene in TNBC mouse models. Mice received a daily oral treatment with different doses of raloxifene. Tumor progression was monitored weekly; in addition microvessel density, proliferation, migration and invasion, apoptosis and tumorigenicity were analyzed. This study demonstrates that raloxifene $(0.85 \mathrm{mg} / \mathrm{kg})$ prevents TNBC tumor growth and induces tumor regression. The treated tumors showed a $54 \%$ decreased microvascular density and proliferation and a 7-fold increase in apoptosis. The underlying therapeutic mechanism of raloxifene was associated with a 27-fold decrease in the expression of the epidermal growth factor receptor (EGFR). Moreover, raloxifene promoted the translocation of EGFR into endosomes associated with decreased cell migration, cell invasion and tumorigenicity in vitro. Together, these data showed that raloxifene acts independently of the ER and may be relevant for the treatment as well as control the progression of TNBC.
\end{abstract}

\section{Introduction}

Selective estrogen receptor modulators (SERMs) such as tamoxifen and raloxifene have proven to be successful in the treatment of breast cancer. Raloxifene, a second generation SERM, has been approved for the prevention of osteoporosis and the reduction of the risk of invasive breast cancer in postmenopausal women (1). In breast tissue, SERMs are thought to prevent proliferation of cancer cells by binding competitively to the estrogen receptor (ER) and blocking the mitogenic

Correspondence to: Dr Sebastien Taurin, Department of Pharmacology and Toxicology, University of Otago, Adams Building, 3rd floor, 18 Frederick Street, Dunedin 9016, New Zealand

E-mail: sebastien.taurin@otago.ac.nz

Key words: selective estrogen receptor modulator, raloxifene, breast cancer, estrogen receptor negative, epidermal growth factor receptor effect of estradiol (2). Although SERMs have a widespread clinical use, it is not established whether their therapeutic effects are solely mediated through the ER. Several studies have demonstrated that SERMs are effective against tumors that do not express ER such as lung cancer (3), brain cancer (4), melanoma (5) and breast cancer (6,7). Furthermore, SERMs have been demonstrated in vitro to trigger multiple signaling pathways that lead to ER-independent mediated cell death (reviewed in ref. 8).

Based on these earlier findings, we investigated the suppressive effects of raloxifene on triple-negative breast cancer (TNBC) growth. By definition, TNBC do not express $\mathrm{ER} \alpha$, progesterone receptor (PR) and human epidermal growth factor receptor 2 (Her2, ErbB2). They account for $10-17 \%$ of all breast cancers and represent $85 \%$ of the basallike subtype (9). TNBCs generally have a higher prevalence in African-American women and an increased occurrence in premenopausal women (9). TNBCs are clinically aggressive and generally associated with a poor prognosis. Currently, chemotherapy remains the only systemic treatment option available for patients with TNBC (10).

In the present study, a daily oral dose of raloxifene not only suppressed tumor growth in two TNBC xenograft mouse models but also promoted tumor regression. The underlying therapeutic mechanism of raloxifene was associated with a decreased expression of EGFR which concurred with a decrease in cell proliferation, an increased incidence of apoptosis and a consequent decrease in blood vessels count within the tumors. In vitro experiments demonstrated that raloxifene decreased EGFR expression by promoting its endocytosis and translocation to small cytoplasmic vesicles akin to those of the endosomal pathway. In addition, raloxifene treatment reduced the migration, invasion and tumorigenicity of MDA-MB-231, a highly metastatic TNBC. Overall these data clearly showed that exploiting the ER-independent mechanisms of raloxifene can promote new therapeutic approaches against TNBC.

\section{Materials and methods}

Cell culture. MDA-MB-231, MDA-MB-468 and MCF-7 were obtained from American Type Culture Collection (ATCC) (Manassas, VA, USA). The cells were grown in complete growth media composed of DMEM/Ham's F12 supplemented with $5 \%$ fetal bovine serum, $2 \mathrm{mM}$ L-glutamine, $100 \mathrm{U} / \mathrm{ml}$ streptomycin, $100 \mathrm{U} / \mathrm{ml}$ penicillin and $2.2 \mathrm{~g} / 1 \mathrm{NaHCO}_{3}$. 
Animals and treatments. All animal protocols were approved by the University of Otago Animal Ethics Committee (\#91/07x2). Female CD1 athymic nude mice (5-6-week-old) were purchased from Hercus Taieri Resource Unit (Dunedin, New Zealand). Mice were inoculated subcutaneously into the right rear flank with triple-negative cells either MDA-MB-231 (2×106 cells/0.1 ml Matrigel) or MDA-MB-468 (8x10 ${ }^{6}$ cells $/ 0.2 \mathrm{ml}$ Matrigel). When tumors reached a size of $\sim 100 \mathrm{~mm}^{3}$ (MDA-MB-231 cells), $200 \mathrm{~mm}^{3}$ (MDA-MB-468 cells) or $400-500 \mathrm{~mm}^{3}$ (MDA-MB-468 cells for analyzing tumor regression), six animals were randomly assigned per treatment groups. Daily for 8-10 weeks as specified, mice received either raloxifene $(0.5 \mathrm{mg} / \mathrm{kg})$, raloxifene $(0.85 \mathrm{mg} / \mathrm{kg})$, raloxifene $(12.5 \mathrm{mg} / \mathrm{kg})$ or a vehicle control (0.25\% DMSO). Two independent measurements of tumor volume (length $\mathrm{x}$ width $\mathrm{x}$ height) were performed weekly using electronic calipers.

Western blot analysis, immunohistochemistry and indirect immunofluorescence. Tissues and MDA-MB-468 cells were processed as described (11) and western blot analysis was performed either with EGFR antibody (Cell Signaling, Danvers, MA, USA), $\beta$-actin (Sigma-Aldrich, Auckland, New Zealand) or ER $\alpha$ (Abcam, Cambridge, MA, USA). MCF-7 cell lysates were used as a positive control for ER $\alpha$ expression. For immunohistochemistry, frozen sections, obtained from tumors were embedded in OCT, then incubated overnight with either rat anti-mouse CD105 (BD Pharmingen, Auckland, New Zealand) or rabbit Ki67 (Epitomics, Burlingame, CA, USA). Slides were incubated with the appropriate biotinylated secondary antibody either goat anti-rat (BD Pharmingen) or goat anti-rabbit (Dako, Campbellfield, Australia). The sections were then incubated with streptavidin (BD Pharmingen) before development with 3,3'-diaminobenzidine tetrahydrochloride (DAB) (BD Pharmingen) and counterstained with hematoxylin QS (Vector Laboratories). In situ labeling of fragmented DNA, TUNEL assay (terminal deoxynucleotidyl transferase-mediated 2'-deoxyuridine 5'-triphosphate nick-end labeling) was carried out using the apotag peroxidase In Situ Apoptosis Detection kit (Millipore, North Ryde, Australia) according to the manufacturer's instructions. Indirect immunofluorescence microscopy was carried out as described previously (12). Briefly, cells were incubated with raloxifene for $48 \mathrm{~h}$, fixed with $4 \%$ paraformaldehyde and incubated with EGFR antibody alone or in combination with EEA1 or caveolin-1 antibodies (Cell Signalling). Secondary antibodies, conjugated to fluorescein or Texas Red (Dako), were used for co-localization. Nuclei were visualized using 4',6-diamidino2-phenylindole (DAPI) staining.

Cell migration. Migration of MDA-MB-231 cells was measured with the in vitro cell scratch assay. Confluent cells were scratched with a pipette tip and cellular debris were removed by extensive washing with serum-free medium. Raloxifene $(10 \mu \mathrm{M})$ or DMSO as control was then added. Cells were allowed to migrate into the scrapped area for up to $20 \mathrm{~h}$ at $37^{\circ} \mathrm{C}$ and were captured at indicated intervals.

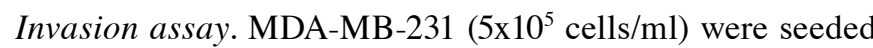
onto growth factor-reduced Matrigel invasion chambers $(8-\mu \mathrm{m}$ pore; BD Biosciences) with or without raloxifene $10 \mu \mathrm{M}$ for 20 h. Lower chambers contained DMEM/Ham's F12 supplemented with $5 \%$ FBS, a chemoattractant. Filters were fixed in methanol and stained using Diff-Quick staining solutions. Cells were counted in four fields of each well under an inverted microscope at magnification, $\mathrm{x} 20$. Their migration towards FBS was calculated as a percentage of the control. Data were collected from three independent experiments, each done in triplicate. Migrated cells were counted and the mean $( \pm$ SE) between groups were analyzed using a Student's t-test.

Soft-agar assay. The base layer consisted of $0.6 \%$ ultra-pure agarose (Invitrogen, Auckland, New Zealand) in DMEM/ Ham's F12 supplemented with 5\% FBS medium. Soft agar composed of $0.3 \%$ ultra-pure agarose in DMEM/Ham's F12 supplemented with 5\% FBS medium was mixed with $15 \times 10^{4}$ MDA-MB-231 cells and plated on top of the solidified base layer in a 6-well-plate. Soft agar cultures were maintained at $37^{\circ} \mathrm{C}$ for an additional 21 days and treated with raloxifene at the indicated concentration $(5,10$ or $15 \mu \mathrm{M})$ or DMSO $(0.1 \%)$. Formed colonies were stained with $0.2 \%$ (w/v) crystal violet (Sigma-Aldrich) solution in 6\% (v/v) paraformaldehyde solution (Sigma-Aldrich). Colonies were counted in images taken in four fields in each well. The assay was repeated three times with duplicate samples.

Statistical analysis. Before statistical analysis, data were log-transformed if parameters showed significantly different variances between control and treated mice (namely, tumor volume and tumor weight). Tumor growth experiments were analyzed using a repeated measures two-way ANOVA coupled with a Student-Newman-Keuls post hoc test, where $\mathrm{p}<0.05$ is required for statistical significance. Analyses that were independent of time (i.e., tumor weight and protein expression) were analyzed using a one-way ANOVA coupled with a Student-Newman-Keuls post hoc test, where $\mathrm{p}<0.05$ is required for statistical significance.

\section{Results}

A daily oral dose of raloxifene suppresses tumor growth. Tumor growth was abolished in two mouse models by a daily administration of raloxifene with optimal dose being $0.85 \mathrm{mg} /$ $\mathrm{kg}$ (Fig. 1). In the first model, MDA-MB-231 xenograft tumors were seeded in mice until reaching a size of $100 \pm 12 \mathrm{~mm}^{3}$. The doses of raloxifene used in this experiment were 25 and 15 times lower than the human equivalent dose of $60 \mathrm{mg}$ when calculated based on body surface area (13). The results showed that after 4 weeks, the mice receiving raloxifene daily showed a significant reduction in tumor growth compared to vehicle control (Fig. 1A). At 8 weeks, these raloxifene treated groups showed tumors sized at $\sim 100 \mathrm{~mm}^{3}, 10$ times lower than those of the vehicle group $\left(\sim 1,000 \mathrm{~mm}^{3}\right)(\mathrm{p}<0.001)$ and a similar size comparable to those before the start of the treatment. In the second model, the rear flanks of mice were implanted with MDA-MB-468 cells. The doses of raloxifene used were either 15 times lower or equivalent to the human dose of $60 \mathrm{mg}$. The results showed that after 10 weeks of treatment, tumor size for both raloxifene treated groups were three times lower than that of the vehicle treated group $\left(450 \mathrm{~mm}^{3}\right)(\mathrm{p}<0.001)$. 
A
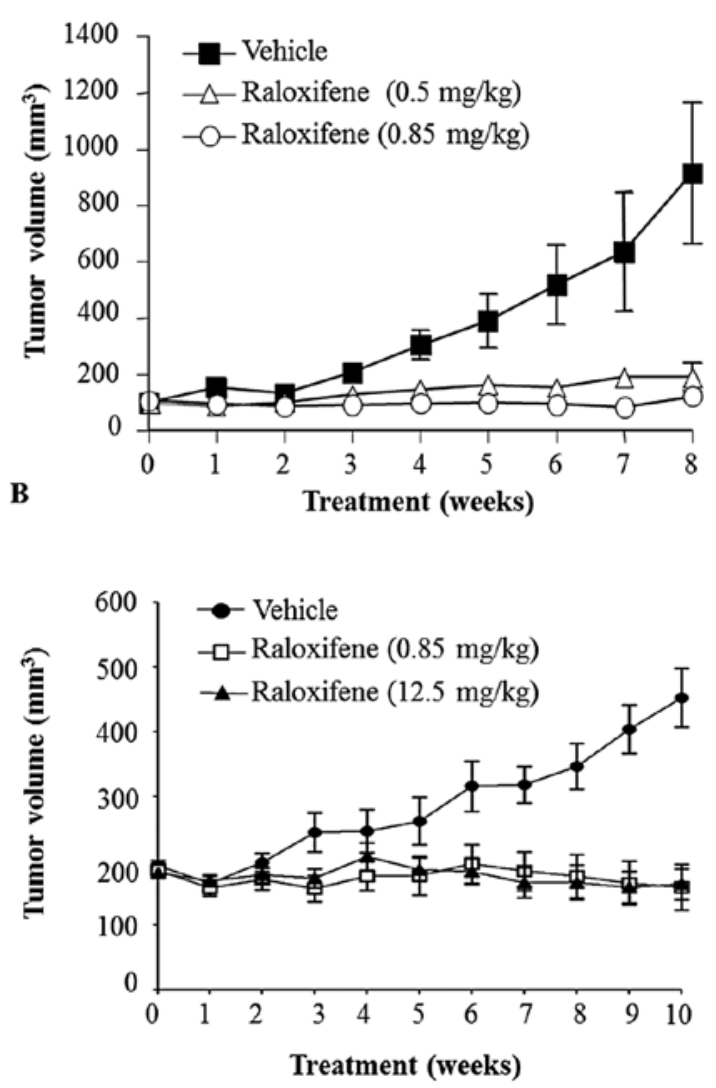

Figure 1. A low oral dose of raloxifene prevents tumor growth of MDAMB-231 cells and MDA-MB-468 cell xenograft models. (A) Female athymic nude mice were implanted with MDA-MB-231 cells $\left(2 \times 10^{6}\right)$ and treated for 56 days with either vehicle or raloxifene $(0.5$ or $0.85 \mathrm{mg} / \mathrm{kg}$, p.o.). (B) Female athymic nude mice were implanted with MDA-MB- 468 cells $\left(8 \times 10^{6}\right)$ and treated for 70 days with either vehicle or raloxifene $(0.85$ or $12.5 \mathrm{mg} / \mathrm{kg}$, p.o.). Tumor volume ( $\mathrm{LxWxH})$ was measured weekly by two independent examiners using an electronic caliper. Symbols represent the mean \pm SEM from 6 mice. Data were analyzed using repeated measures two-way ANOVA coupled with the Student-Newman-Keuls post hoc test. $p<0.05$ was observed after 5 weeks in each treatment model.

Interestingly, the low dose of raloxifene $(0.85 \mathrm{mg} / \mathrm{kg})$ was as effective at suppressing MDA-MB-468 xenograft tumor growth as the higher $(12.5 \mathrm{mg} / \mathrm{kg})$ human equivalent dose (Fig. 1B). Moreover, in both tumorigenic models, the body weights of animals receiving raloxifene did not change in comparison to controls.

A daily oral dose of raloxifene caused regression of $T N B C$ xenograft tumors. The effective low dose of raloxifene $(0.85 \mathrm{mg} /$ $\mathrm{kg}$ ) promoted tumor volume regression. Female athymic nude mice were implanted with MDA-MB-468 cells. In this model after tumors reached an average volume of $400-500 \mathrm{~mm}^{3}$, the mice were treated daily for a period of 10 weeks with an oral dose of $0.85 \mathrm{mg} / \mathrm{kg}$ of raloxifene, or vehicle control (DMSO (0.25\%) (Fig. 2). After 5 weeks, tumors of raloxifene treated mice were $52 \%$ smaller $(\mathrm{p}<0.05)$ than those of vehicle controls (Fig. 2A). After 10 weeks of treatment, tumor size had decreased to $283 \mathrm{~mm}^{3}$, a $39 \%$ reduction from their initial size and a $70 \%$ volume reduction compared to vehicle controls $(\mathrm{p}<0.001)$ (Fig. 2A). Consequently, tumor weight was 5 times lower in raloxifene treated mice compared to vehicle controls
(Fig. 2B). Immunohistological analysis using CD105 antibody revealed that raloxifene treatment reduced CD105 positive microvascular density by $50 \%$ (Fig. $2 \mathrm{C}$ and D). This reduction in microvasculature correlated with the observed decrease in tumor volume following the 10 weeks of treatment.

Raloxifene treatment reduces EGFR expression, decreases cell proliferation and increases apoptosis in tumors. The EGFR is among the few proteins that can characterize basallike subtype and TNBC by immunohistology and is one of the highest concordant markers. It is expressed in $60 \%$ of basallike tumors (14) and is highly expressed in MDA-MB-468 cells (15). Furthermore, EGFR stimulates cell proliferation, motility and invasion of breast cancer cells $(16,17)$. Western blot analysis showed that raloxifene $(0.85 \mathrm{mg} / \mathrm{kg})$ significantly decreased the expression of EGFR protein (Fig. 3A and B). Treatment of MDA-MB-468 cells with raloxifene $(10 \mu \mathrm{M})$ for $24 \mathrm{~h}$ in vitro also triggered a decreased expression of EGFR by $>30 \%$ (data not shown). We confirmed that long-term in vivo raloxifene treatment did not induce ER $\alpha$ expression in MDA-MB-468 tumors (Fig. 3A). Raloxifene therapy significantly inhibited tumor cell proliferation, as shown by a $70 \%$ decrease in cells positive for Ki67 compared to vehicle controls (Fig. 3C). Moreover, raloxifene treatment induced an 8-fold elevation of the number of TUNEL-positive cells compared to the vehicle group (Fig. 3D and E).

Raloxifene treatment promotes EGFR endocytosis. Raloxifene treatment of MDA-MB-468 cells in vitro changes EGFR localization and promotes its transit towards small cytoplasmic vesicles. In untreated MDA-MB-468 cells, EGFR is highly expressed and localizes at the membrane but also shows a diffuse punctuate cytoplasmic staining (Fig. 4A). Whereas, after 48 -h exposure to raloxifene $(10 \mu \mathrm{M})$ triggers the accumulation of EGFR in small cytoplasmic vesicles (Fig. 4B). To establish the origin of the cytoplasmic vesicles containing EGFR, we probed two protein markers: early endosome antigen-1 (EEA1), which is essential for early endosome formation and trafficking (18) and caveolin-1, a marker of caveolae and endosome formation (19). In control cells, dual labeling with EEA1 and EGFR showed that these proteins distinctively compartmentalize (Fig. 4A) where EEA1 is cytoplasmic but can also cluster within small vesicles while EGFR is mainly expressed at the cell membrane. However, in raloxifene treated cells, the two proteins colocalized within a few larger vesicles (Fig. 4B), while some vesicles contained only EGFR protein (Fig. 4B). When probing raloxifene treated cells with EGFR and caveolae marker, we found that caveolin-1 and EGFR antibodies colocalized both proteins within a few vesicles (Fig. 4D) while in the untreated control cells, the caveolin-1 staining diffuse (Fig. 4C). Western blot analysis of MDA-MB-468 cells showed that raloxifene treatment decreased EGFR phosphorylation and protein expression after a 24-h incubation and this was associated with a reduced expression of downstream effectors such as NFkB (Fig. 4E). Overall, these experiments show that the EGFR is internalized into cytoplasmic vesicles related to endosome formation. This suggests that the raloxifene mediated decrease in proliferation of cancer cells may be mediated through endocytosis of the EGFR which decreases proliferative signaling pathways. 
$\mathbf{A}$

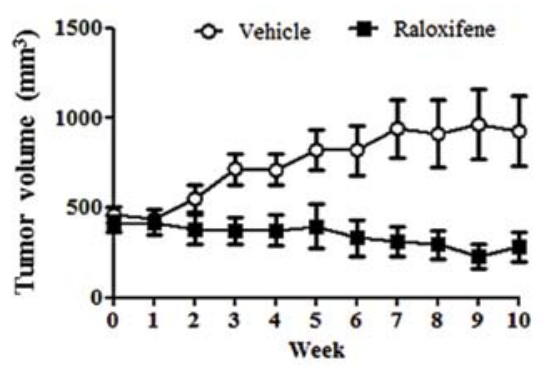

B

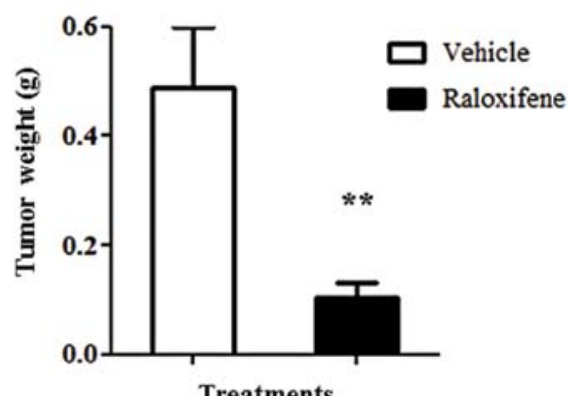

C

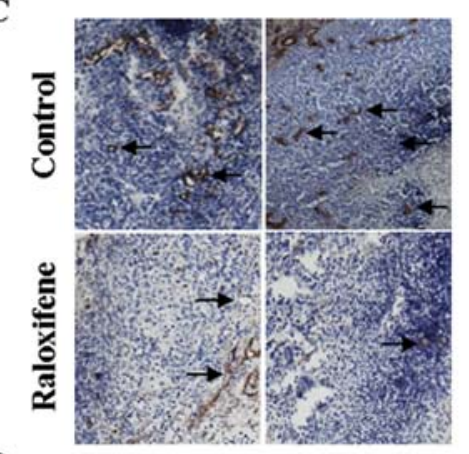

D

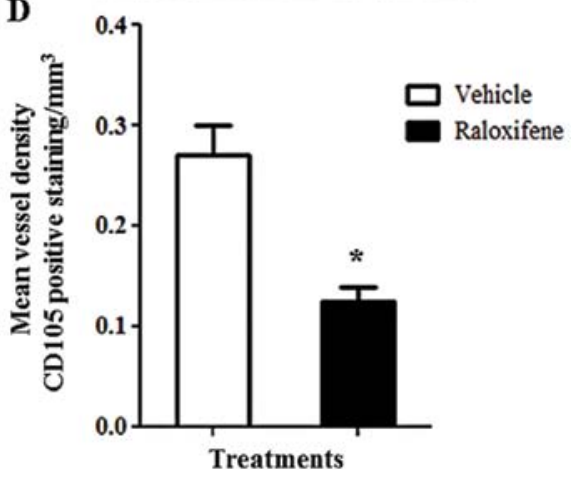

Figure 2. A low oral dose of raloxifene causes TNBC regression in a xenograft model. Female athymic nude mice were implanted with MDA-MB-468 cells $\left(8 \times 10^{6}\right)$ and treated for 70 days with either vehicle (DMSO $0.25 \%$, p.o.) or raloxifene $(0.85 \mathrm{mg} / \mathrm{kg}$, p.o.). (A) Tumor volume (LxWxH) was measured weekly using electronic calipers. Symbols represent the mean \pm SEM from 6 mice. Data were analyzed using a repeated measure two-way ANOVA coupled with the Student-Newman-Keuls post hoc test. The p-value was p<0.05 after 5 weeks of treatment. (B) Tumor weight after 70 days of treatment with vehicle or raloxifene $\left(0.85 \mathrm{mg} / \mathrm{kg}\right.$, p.o.). Columns represent the mean \pm SEM from 6 mice per group; ${ }^{* *} \mathrm{p}<0.01$. (C) CD105 expression was assessed by immunohistochemistry of tissue sections obtained from tumors from both treatment groups as a representation of the mean vessel density. (D) The histogram shows the mean vessel density \pm SEM from both groups; " $<<0.05$.

A

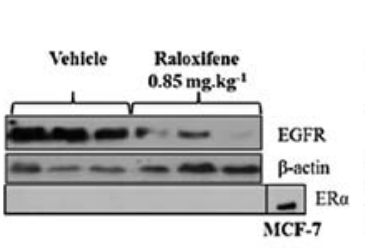

B

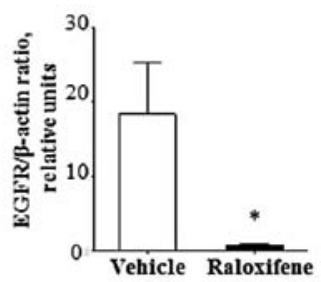

C

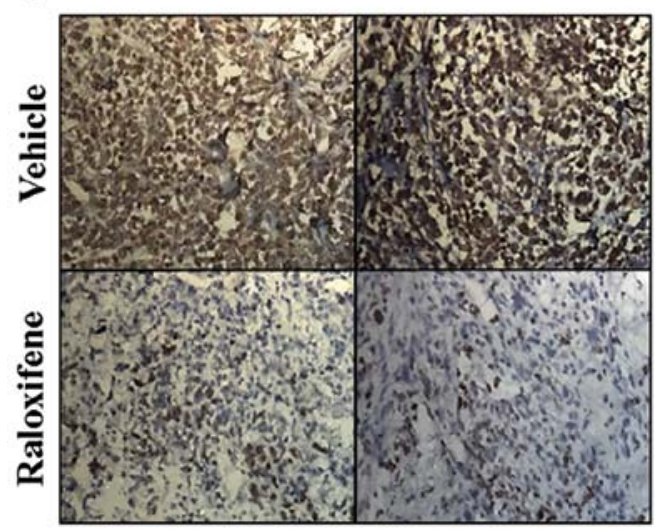

D

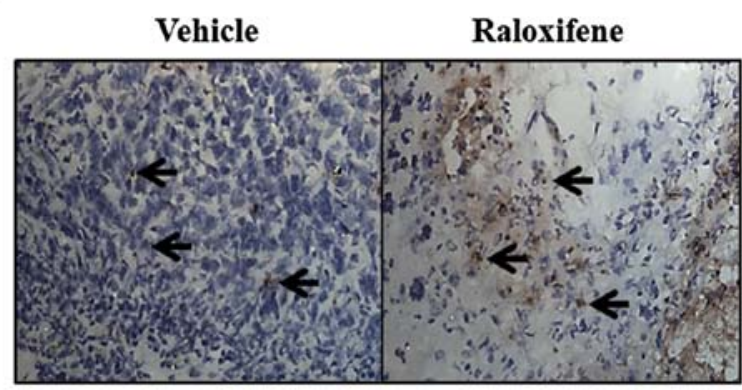

$\mathbf{E}$

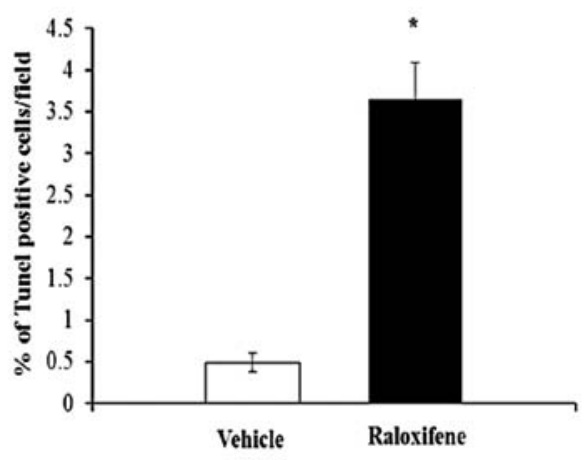

Figure 3. Raloxifene treatment decreases EGFR expression, cell proliferation and induces apoptosis in TNBC xenograft tumors. (A) Representative western blots of EGFR and ER $\alpha$ protein expression in tumors. MCF-7 cell lysate was used as a positive control for ER $\alpha$ expression. (B) EGFR densitometry obtained from 6 mice from each treatment group. Data were analyzed using the Mann-Whitney U test in which $\mathrm{p}<0.05$ denoted a statistically significant difference. (C) Immunohistochemical staining of Ki67 in tissue sections of xenograft tumors obtained from vehicle and raloxifene treatment groups. Original magnification, $x 400$. (D) TUNEL assay based immunohistochemical staining of apoptotic cells in tissue sections of xenograft tumor obtained from both treatment groups. Original magnification, $x 400$. The arrows indicate positive TUNEL staining. (E) Quantification of apoptosis following TUNEL staining. Results are expressed as mean \pm SEM of six 400X fields $(\mathrm{p}<0.05)$. 
A

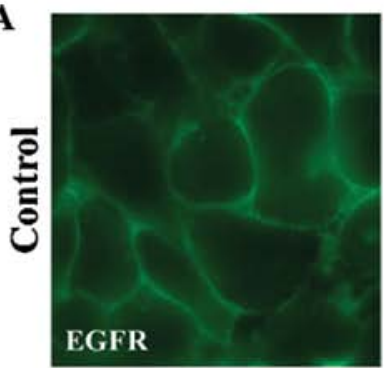

B

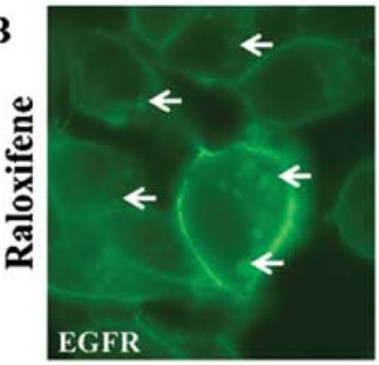

C

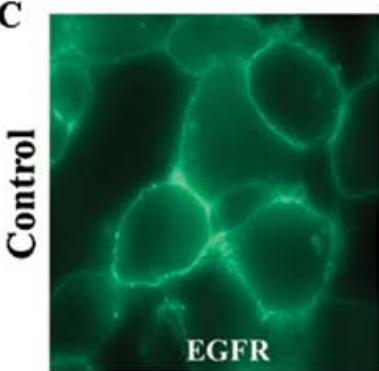

D

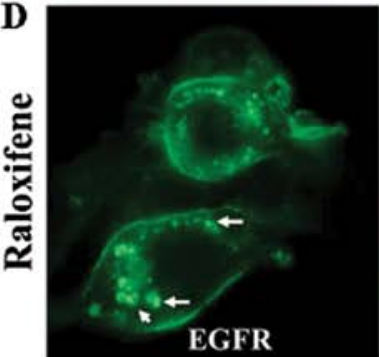

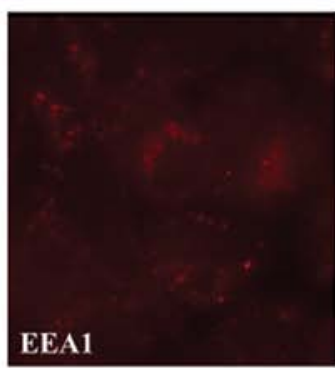
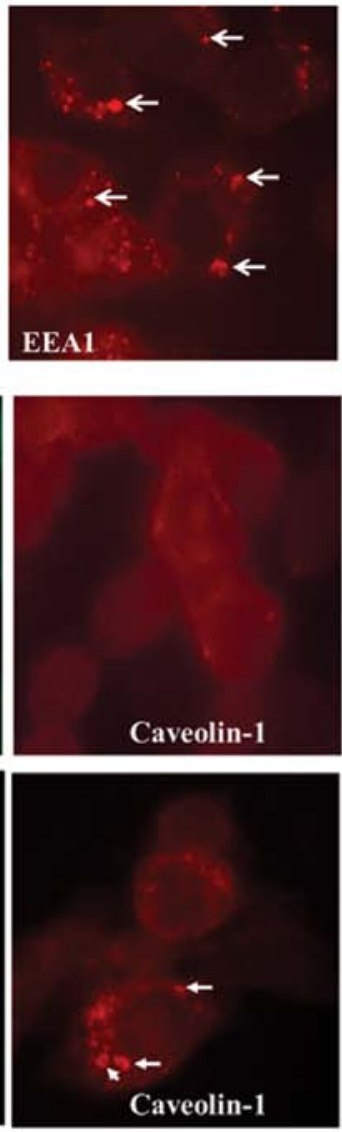
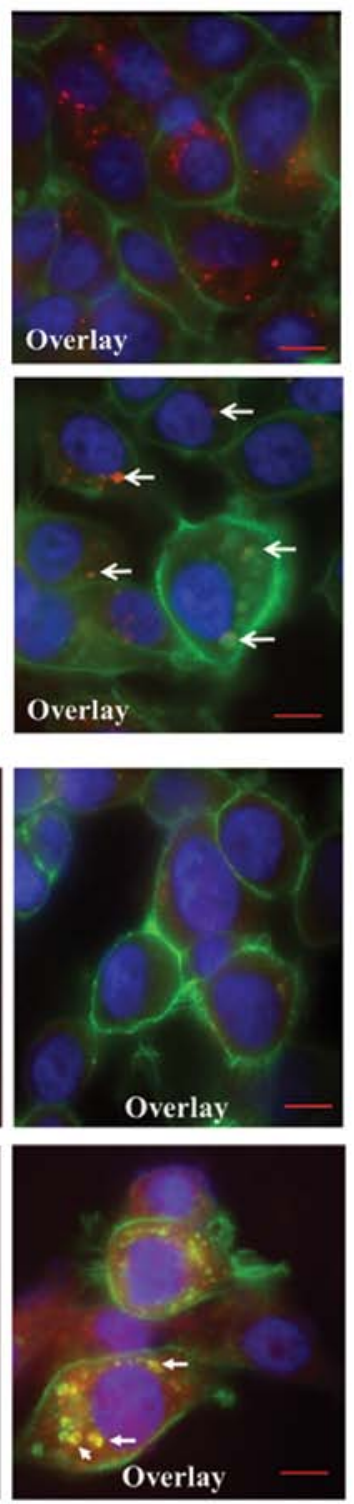

$\mathbf{E}$

$\begin{array}{llllllllll}0 & 0.5 & 1 & 3 & 6 & 12 & 24 & 36 & 48 & \text { Time (h) }\end{array}$

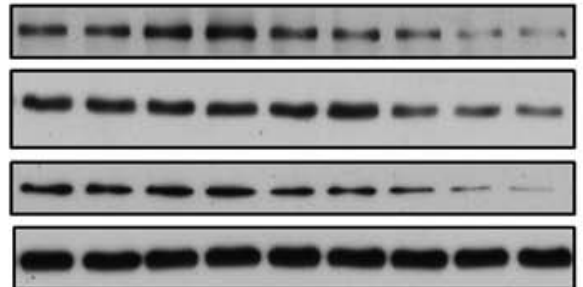

P-EGFR

EGFR

$\mathrm{NF} \kappa \mathrm{B}$

$\beta$-tubulin

Figure 4. Raloxifene promotes accumulation of EGFR in small cytoplasmic vesicles associated with the endosomal pathway. MDA-MB-468 cells were treated with vehicle (DMSO $0.1 \%$ ) or raloxifene $(10 \mu \mathrm{M})$ for $48 \mathrm{~h}$. (A) Co-immunofluorescence of EGFR and EEA1 proteins shows no colocalization of the proteins in untreated control cells. (B) While in the raloxifene treated cells, EGFR and EEA1 colocalized in a few vesicles. Arrows indicated vesicles showing EGFR co-localization with EEA1. (C) Immunofluorescence of EGFR and caveolin-1 proteins shows a diffuse pattern of caveolin-1 and no vesicle formation in untreated control cells. (D) In the raloxifene treated cells, EGFR and caveolin-1 are present in the same vesicles. The arrows indicate vesicle containing both proteins. Original magnification, x2,000 (scale bar indicates the length of $10 \mathrm{~nm}$ ). (E) Western blot of phospho-EGFR (Tyr-1148), EGFR and NFkB proteins of MDA-MB-468 cells treated with raloxifene $(10 \mu \mathrm{M})$ up to $48 \mathrm{~h}$.

Raloxifene decreases tumorigenicity, cell migration and cell invasion. Raloxifene treatment significantly decreased anchorage-independent growth of the highly metastatic MDA-MB-231 cells in soft agar. The assay we used represents an in vitro transformation phenotype that is highly correlated with in vivo tumorigenicity (20). Raloxifene not only dose- dependently suppressed colony formation of MDA-MB-231 cells (Fig. 5A and B) but treatment decreased their cell migration and invasion. Specifically, raloxifene $(10 \mu \mathrm{M})$ reduced cell migration and delayed wound closure by $70 \%$ (Fig. 5C and D). The ability of raloxifene to prevent MDA-MB-231 cells to migrate and invade through Matrigel was then measured in 
A

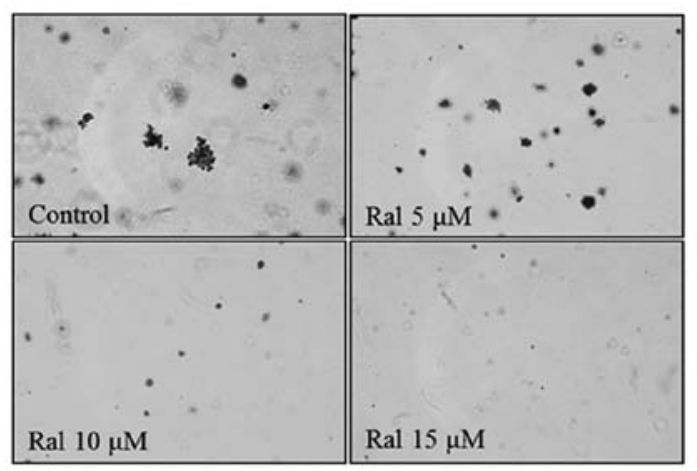

C

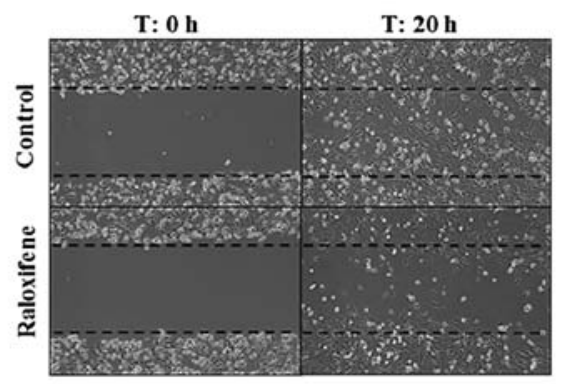

$\mathbf{E}$

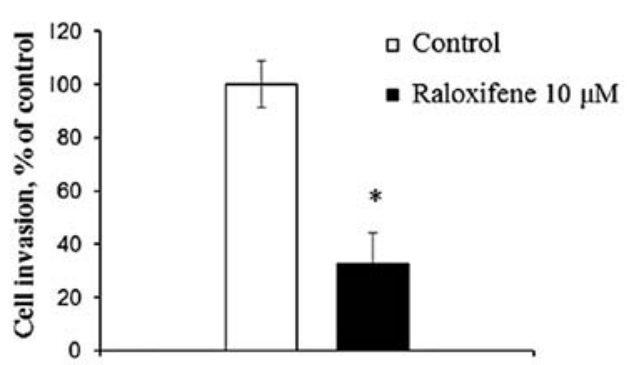

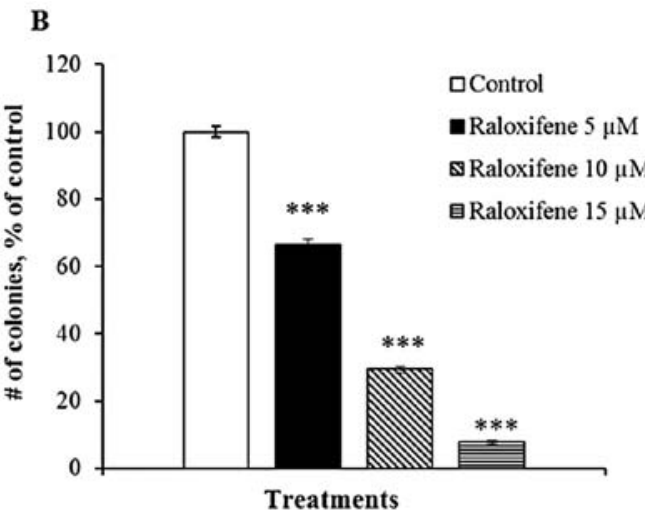

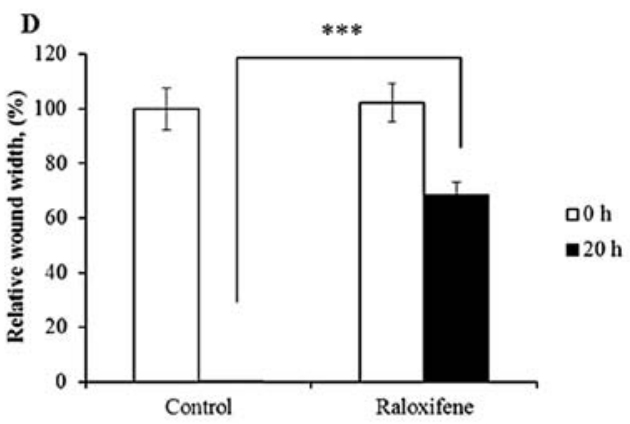

Figure 5. Raloxifene treatment decreases tumorigenicity, cell migration and invasion in vitro. (A) MDA-MB-231 cells were plated in soft agar, treated with either DMSO $0.1 \%$ or raloxifene $(5,10$ or $15 \mu \mathrm{M})$. The colonies were counted after 21 days. (B) The histogram represents the number of colonies normalized to the control treated cells; ${ }^{* * *} \mathrm{p}<0.001$. (C) A confluent MDA-MB-231 monolayer was wounded and incubated for $20 \mathrm{~h}$ with either DMSO $0.1 \%$ or raloxifene $(10 \mu \mathrm{M})$. Representative monolayer images are shown at 0 and $20 \mathrm{~h}$. (D) The histogram represents the wound width after 20 -h incubation; ${ }^{* * *}$ p $<0.001$. (E) MDA-MB-231 cells were seeded onto filters precoated with Matrigel on the upper surface in transwell chambers and treated with either 0.1\% DMSO or raloxifene $(10 \mu \mathrm{M})$. The bottom chamber contained growth media with $5 \%$ fetal bovine serum as the chemoattractant. After $20 \mathrm{~h}$ the cells migrating to the lower surface were fixed and stained with Diff Quick. Bars represent the mean \pm SEM of three independent experiments; ${ }^{p}$ p $<0.05$.

a transwell chamber. Raloxifene impaired invasion by $>70 \%$ compared to control (Fig. 5E). Together these in vitro results provide further evidence that raloxifene significantly reduces the metastatic potential of TNBC cells.

\section{Discussion}

Despite the high rate of response to chemotherapy, patients presenting TNBC have considerably poor prognosis. This urges the development of novel targeted or combination therapies to reduce the mortality associated with these cancers. By identifying the targets and mechanisms behind the efficacy of a drug helps to carefully adapt treatment conditions to specific pathology of TNBC. The present study describes four major findings: i) a daily oral dose of raloxifene suppresses tumor growth in two xenograft mouse models of TNBC; ii) a daily oral dose of raloxifene promotes tumor regression in a xenograft model using MDA-MB-468 cells; iii) raloxifene treatment decreases the expression of EGFR and promotes its accumulation in endosomes; iv) raloxifene decreases tumorigenicity, cell migration and invasion of a highly invasive human TNBC cell line.

The efficacy of SERMs, tamoxifen and raloxifene, has been attributed to their ability to compete with $17 \beta$-estradiol antagonizing ER downstream signaling events (21). Several studies have demonstrated the in vivo and in vitro proapoptotic potential of SERMs in various ER-negative tissues and cells including bladder, glioma, melanoma or breast cancer (1,22-24). The in vitro effect of the SERMs was concentration-dependent, as growth arrest was induced by nanomolar concentrations while cell death was achieved in the micromolar range (1). In addition to the specific antagonistic effects of SERMs in the breast, other data suggest antitumorigenic effect of SERMs that are independent of ER $\alpha$ signaling. For example, raloxifene was shown to modulate phospholipase D activity (25) and more recently both raloxifene and tamoxifen were shown to reduce glutamine uptake (26). 
In our study, we observed that raloxifene affects the expression of EGFR, a protein known to have a prominent role in the development of TNBC. EGFR expression is found in 45-70 of TNBC (27) and is a putative biomarker associated with an unfavorable prognosis (28). Analysis by western blot analysis showed that EGFR expression in tumors was decreased by a daily dose of raloxifene. The present study suggests that its effects on cell proliferation and apoptosis would stem from a decrease in EGFR and its clustering within endosomes. Furthermore, previous studies have demonstrated that internalized EGFR can promote caspase-3 mediated apoptosis (29).

Interestingly, SERMs such as raloxifene and tamoxifen were shown to bind with high affinity to the microsomal antiestrogen binding site (AEBS) (30). AEBS is a protein complex composed of two enzymes and acts as a cholesterol epoxyde hydrolase, an enzyme involved in cholesterol metabolism (31). AEBS has no affinity for estrogens and in the MDA-MB-468 cells, an ER $\alpha$-negative breast cancer cell line, it is highly expressed in comparison to the ER $\alpha$-positive cell line, MCF-7 (32). Recently, a study demonstrated that tamoxifen binding to AEBS induces the formation of small cytoplasmic vesicles or phagosomes in MCF-7 cells and their accumulation promoted cellular apoptosis (30). Few of these phagosomes contain the early endocytic marker EEA1 (33). These vesicles can fuse with endosomes leading to the degradation of their content (34). Moreover, caveolae vesicles have been shown to mediate the internalization of proteins at the plasma membrane into cells, such as the EGFR (35). Internalization of EGFR could follow two distinct endocytotic routes: the clathrin-dependent one and the clathrin-independent route mediated by caveolin (36). The EGFR-caveolin interaction reduces the activation of EGFR signaling (37). Furthermore, a recent study demonstrated that the accumulation of EGFR in the endosomes of MDA-MB-468 cells induced EGFR-mediated apoptosis (15).

However, the effects of raloxifene on EGFR expression and localization could be explained by another potential mechanism routed in the possible ability of ER $\beta$ to control EGFR signaling. Expression of ER $\beta$ has been detected in $20 \%$ of TNBC (38) and at lower level in MDA-MB-468 cells (39). However, its role, if any, in the development and progression of breast cancer remains unclear. Raloxifene binds with high affinity to ER $\alpha$ and ER $\beta$ and usually acts as an antagonist in the breast (40). Interestingly, a recent study has identified a physical interaction between ER $\beta$, EGFR and caveolin-1 which reduces EGFR signaling in lung cancer (41), a similar mechanism involving raloxifene binding to ER $\beta$ and the triggering of EGFR endocytosis may be responsible for the tumor suppressing effect of raloxifene treatment in vivo and warrants further analysis.

Several studies have identified a truncated variant of $36-\mathrm{kDa}$ of $\mathrm{ER} \alpha(\mathrm{ER} \alpha-36)$, which is frequently expressed in TNBC (42). ER $\alpha-36$ has no intrinsic transcriptional activity (43) but mediates non-genomic estrogen signaling through the EGFR/Src/ERK signaling pathway in TNBC (44). ER $\alpha-36$ was shown to possibly enhance tamoxifen agonist activity in endometrial cancer cell lines suggesting the capability of SERMs to directly bind to ER $\alpha-36$ (45). Therefore, the effect of raloxifene could be mediated through ER $\alpha-36$ interaction leading to a decreased EGFR expression and its downstream signaling pathways.
The majority of cancer-related deaths are caused by metastasis, a multistep process that depends on alterations of tumor microenvironment, survival of cancer cells in the circulation and colonization of a distant organ (46). Previous studies have shown that in TNBC, a low level of EGFR expression correlates with a reduced incidence of metastases (47). Inhibition of invasive potential is important for the prevention of tumor recurrence. Raloxifene treatment effectively reduced migration, invasion and the malignancy potential of MDA-MB-231 cells in vitro and reduced microvessel density in vivo. However, further in vivo studies are needed to definitively prove the potential of raloxifene alone or in combination to prevent $\mathrm{ER} \alpha$-negative metastasis.

Collectively these data show that an oral daily dose of raloxifene suppressed tumor growth in two relevant mouse xenograft models of TNBC. Moreover, raloxifene treatment acted independently of ER $\alpha$ and this was mediated by decreased EGFR protein levels and their altered localizations within endosomes. Overall, this study shows that raloxifene can be a valuable treatment for TNBC and significant new targets can be identified with further studies. This mechanistic information may lead to the development of new therapies for TNBC.

\section{Acknowledgements}

This study was supported by grants from the Otago Medical Research Foundation (AG302-ST), UORG (ST), Health Research Council of New Zealand (ST) and Lottery Health (ST). We would like to thank Ms. Hayley Nehoff and Ms. Céline Bourdon for the editorial assistance.

\section{References}

1. Sporn MB, Dowsett SA, Mershon J and Bryant HU: Role of raloxifene in breast cancer prevention in postmenopausal women: clinical evidence and potential mechanisms of action. Clin Ther 26: 830-840, 2004

2. Jordan VC and Koerner S: Inhibition of oestradiol binding to mouse uterine and vaginal oestrogen receptors by triphenylethylenes. J Endocrinol 64: 193-194, 1975.

3. Croxtall JD, Emmas C, White JO, Choudhary Q and Flower RJ: Tamoxifen inhibits growth of oestrogen receptor-negative A549 cells. Biochem Pharmacol 47: 197-202, 1994.

4. Couldwell WT, Weiss MH, DeGiorgio CM, et al: Clinical and radiographic response in a minority of patients with recurrent malignant gliomas treated with high-dose tamoxifen. Neurosurgery 32: 485-490, 1993.

5. Del Prete SA, Maurer LH, O'Donnell J, Forcier RJ and LeMarbre P: Combination chemotherapy with cisplatin, carmustine, dacarbazine and tamoxifen in metastatic melanoma. Cancer Treat Rep 68: 1403-1405, 1984.

6. Murphy LC and Sutherland RL: Differential effects of tamoxifen and analogs with nonbasic side chains on cell proliferation in vitro. Endocrinology 116: 1071-1078, 1985.

7. Plowman PN: Tamoxifen as adjuvant therapy in breast cancer. Current status. Drugs 46: 819-833, 1993.

8. Mandlekar S and Kong ANT: Mechanisms of tamoxifen-induced apoptosis. Apoptosis 6: 469-477, 2001.

9. Carey LA, Dees EC, Sawyer L, et al: The triple-negative paradox: primary tumor chemosensitivity of breast cancer subtypes. Clin Cancer Res 13: 2329-2334, 2007.

10. Kaplan HG and Malmgren JA: Impact of triple-negative phenotype on breast cancer prognosis. Breast J 14: 456-463, 2008.

11. Somers-Edgar TJ, Taurin S, Larsen L, Chandramouli A, Nelson MA and Rosengren RJ: Mechanisms for the activity of heterocyclic cyclohexanone curcumin derivatives in estrogen receptor negative human breast cancer cell lines. Invest New Drugs 29: 87-97, 2011 
12. Taurin S, Sandbo N, Qin Y, Browning D and Dulin NO Phosphorylation of $\beta$-catenin by cyclic AMP-dependent protein kinase. J Biol Chem 281: 9971-9976, 2006.

13. Reagan-Shaw S, Nihal $M$ and Ahmad N: Dose translation from animal to human studies revisited. FASEB J 22: 659-661, 2008.

14. Nielsen TO, Hsu FD, Jensen K, et al: Immunohistochemical and clinical characterization of the basal-like subtype of invasive breast carcinoma. Clin Cancer Res 10: 5367-5374, 2004.

15. Rush JS, Quinalty LM, Engelman L, Sherry DM and Ceresa BP: Endosomal accumulation of the activated epidermal growth factor receptor (EGFR) induces apoptosis. J Biol Chem 287: 712-722, 2012.

16. Ueno NT and Zhang D: Targeting EGFR in triple-negative breast cancer. J Cancer 2: 324-328, 2011.

17. Nickerson NK, Mohammad KS, Gilmore JL, et al: Decreased autocrine EGFR signaling in metastatic breast cancer cells inhibits tumor growth in bone and mammary fat pad. PLoS One 7: e30255, 2012.

18. Zoncu R, Perera RM, Balkin DM, Pirruccello M, Toomre D and De Camilli P: A phosphoinositide switch controls the maturation and signaling properties of APPL endosomes. Cell 136: $1110-1121,2009$.

19. Pol A, Lu A, Pons M, Peiró S and Enrich C: Epidermal growth factor-mediated caveolin recruitment to early endosomes and MAPK activation. J Biol Chem 275: 30566-30572, 2000.

20. Freedman VH and Shin SI: Cellular tumorigenicity in nude mice: correlation with cell growth in semi-solid medium. Cell 3: 355-359, 1974

21. Riggs BL and Hartmann LC: Selective estrogen-receptor modulators - mechanisms of action and application to clinical practice N Engl J Med 348: 618-629, 2003.

22. Perry RR, Kang Y and Greaves B: Effects of tamoxifen on growth and apoptosis of estrogen-dependent and -independent human breast cancer cells. Ann Surg Oncol 2: 238-245, 1995.

23. Gelmann EP: Tamoxifen induction of apoptosis in estrogen receptor-negative cancers: new tricks for an old dog? J Natl Cancer Inst 88: 224-226, 1996.

24. Kim HT, Kim BC, Kim IY, et al: Raloxifene, a mixed estrogen agonist/antagonist, induces apoptosis through cleavage of BAD in TSU-PR1 human cancer cells. J Biol Chem 277: 32510-32515, 2002.

25. Eisen SF and Brown HA: Selective estrogen receptor (ER) modulators differentially regulate phospholipase D catalytic activity in ER-negative breast cancer cells. Mol Pharmacol 62: 911-920, 2002.

26. Todorova VK, Kaufmann Y, Luo S and Klimberg VS: Tamoxifen and raloxifene suppress the proliferation of estrogen receptornegative cells through inhibition of glutamine uptake. Cancer Chemother Pharmacol 67: 285-291, 2011.

27. Bosch A, Eroles P, Zaragoza R, Vina JR and Lluch A: Triplenegative breast cancer: molecular features, pathogenesis, treatment and current lines of research. Cancer Treat Rev 36: 206-215, 2010

28. Cho EY, Chang MH, Choi YL, et al: Potential candidate biomarkers for heterogeneity in triple-negative breast cancer (TNBC). Cancer Chemother Pharmacol 68: 753-761, 2011.

29. Hyatt DC and Ceresa BP: Cellular localization of the activated EGFR determines its effect on cell growth in MDA-MB-468 cells. Exp Cell Res 314: 3415-3425, 2008.

30. de Medina P, Payre B, Boubekeur N, et al: Ligands of the antiestrogen-binding site induce active cell death and autophagy in human breast cancer cells through the modulation of cholesterol metabolism. Cell Death Differ 16: 1372-1384, 2009.
31. de Medina P, Paillasse MR, Segala G, Poirot M and SilventePoirot S: Identification and pharmacological characterization of cholesterol-5,6-epoxide hydrolase as a target for tamoxifen and AEBS ligands. Proc Natl Acad Sci USA 107: 13520-13525, 2010.

32. Payre B, de Medina P, Boubekeur N, et al: Microsomal antiestrogen-binding site ligands induce growth control and differentiation of human breast cancer cells through the modulation of cholesterol metabolism. Mol Cancer Ther 7: 3707-3718, 2008.

33. Simonsen A and Tooze SA: Coordination of membrane events during autophagy by multiple class III PI3-kinase complexes. J Cell Biol 186: 773-782, 2009.

34. Razi M, Chan EY and Tooze SA: Early endosomes and endosomal coatomer are required for autophagy. J Cell Biol 185: 305-321, 2009.

35. Sigismund S, Woelk T, Puri C, et al: Clathrin-independent endocytosis of ubiquitinated cargos. Proc Natl Acad Sci USA 102: 2760-2765, 2005

36. Aguilar RC and Wendland B: Endocytosis of membrane receptors: two pathways are better than one. Proc Natl Acad Sci USA 102: 2679-2680, 2005.

37. Couet J, Sargiacomo M and Lisanti MP: Interaction of a receptor tyrosine kinase, EGF-R, with caveolins. Caveolin binding negatively regulates tyrosine and serine/threonine kinase activities. J Biol Chem 272: 30429-30438, 1997.

38. Litwiniuk MM, Roznowski K, Filas V, et al: Expression of estrogen receptor beta in the breast carcinoma of BRCA1 mutation carriers. BMC Cancer 8: 100, 2008.

39. Vladusic EA, Hornby AE, Guerra-Vladusic FK, Lakins J and Lupu R: Expression and regulation of estrogen receptor beta in human breast tumors and cell lines. Oncol Rep 7: 157-167, 2000.

40. Kuiper GGJM, Lemmen JG, Carlsson B, et al: Interaction of estrogenic chemicals and phytoestrogens with estrogen receptor $\beta$. Endocrinology 139: 4252-4263, 1998.

41. Pinton G, Thomas W, Bellini P, et al: Estrogen receptor $\beta$ exerts tumor repressive functions in human malignant pleural mesothelioma via EGFR inactivation and affects response to gefitinib. PLoS One 5: e14110, 2010.

42. Lee W-L, Chao H-T, Cheng M-H and Wang P-H: Rationale for using raloxifene to prevent both osteoporosis and breast cancer in postmenopausal women. Maturitas 60: 92-107, 2008.

43. Wang Z, Zhang X, Shen P, Loggie BW, Chang Y and Deuel TF: A variant of estrogen receptor- $\alpha$, hER- $\alpha 36$ : transduction of estrogen- and antiestrogen-dependent membrane-initiated mitogenic signaling. Proc Natl Acad Sci USA 103: 9063-9068, 2006.

44. Zhang XT, Kang LG, Ding L, Vranic S, Gatalica Z and Wang ZY: A positive feedback loop of ER-alpha36/EGFR promotes malignant growth of ER-negative breast cancer cells. Oncogene 30: 770-780, 2011.

45. Lin SL, Yan LY, Zhang XT, et al: ER-alpha36, a variant of ER-alpha, promotes tamoxifen agonist action in endometrial cancer cells via the MAPK/ERK and PI3K/Akt pathways. PLoS One 5: e9013, 2010.

46. Nguyen DX, Bos PD and Massague J: Metastasis: from dissemination to organ-specific colonization. Nat Rev Cancer 9: 274-284, 2009.

47. Viale G, Rotmensz N, Maisonneuve P, et al: Invasive ductal carcinoma of the breast with the 'triple-negative' phenotype: prognostic implications of EGFR immunoreactivity. Breast Cancer Res Treat 116: 317-328, 2009. 